\title{
Induction of Hebbian and Non-Hebbian Mossy Fiber Long-Term Potentiation by Distinct Patterns of High-Frequency Stimulation
}

\author{
Nathaniel N. Urban and German Barrionuevo \\ Department of Neuroscience and Center for the Neural Basis of Cognition, University of Pittsburgh, \\ Pittsburgh, Pennsylvania 15260
}

\begin{abstract}
The synapse made by hippocampal mossy fibers onto pyramidal neurons of hippocampal area CA3 displays a form of longterm potentiation (LTP) that is independent of the activation of NMDA receptors. Considerable controversy exists as to whether the induction of mossy fiber LTP requires postsynaptic activation and, thus, whether mossy fiber LTP is Hebbian or non-Hebbian. Here we report the induction of both Hebbian and non-Hebbian forms of long-term potentiation at the mossy fiber-CA3 synapse in in vitro slice preparation. These two forms of potentiation can be induced selectively by different induction
\end{abstract}

Long-term potentiation (LTP) refers to a variety of activitydependent increases in synaptic efficacy relying on several different induction mechanisms (Bramham, 1992; Johnston et al., 1992; Bliss and Collingridge, 1993; Nicoll and Malenka, 1995). LTP requiring coincident activation of pre- and postsynaptic elements is called "Hebbian" LTP, whereas LTP requiring activation of either a pre- or a postsynaptic element is referred to as "nonHebbian" LTP (Hebb, 1949; McNaughton and Barnes, 1990). Postsynaptic NMDA receptors serve as the primary integration site of pre- and postsynaptic activity in most Hebbian forms of plasticity.

LTP at the synapses made by hippocampal mossy fibers onto pyramidal neurons of area $\mathrm{CA} 3$ does not require activation of NMDA receptors (Harris and Cotman, 1986) and thus may be expected to be non-Hebbian. Consistent with this expectation, Zalutsky and Nicoll (1990) reported that activation of mossy fibers can induce LTP at the mossy fiber-CA3 synapse despite blockade of postsynaptic activation by hyperpolarization and buffering of cytosolic calcium. Subsequent studies have confirmed these results (Katsuki et al., 1991; Castillo et al., 1994; Langdon et al., 1995) and, further, have shown that mossy fiber LTP can be induced in the absence of any measurable postsynaptic response (Ito and Sugiyama, 1991; Castillo et al., 1994).

However, in other studies, mossy fiber LTP required both preand postsynaptic activation and, thus, was termed Hebbian (Jaffe and Johnston, 1990; Derrick and Martinez, 1994a). Integration of pre- and postsynaptic activity in NMDA-independent Hebbian LTP may be mediated by voltage-dependent calcium channels (Grover and Teyler, 1992; Johnston et al., 1992) or by cotransmitter systems (Derrick and Martinez, 1994b).

\footnotetext{
Received Feb. 28, 1996; revised April 1, 1996; accepted April 5, 1996.

This work was supported by National Institute of Neurological Disorders and Stroke Grant NS24288, National Institute of Mental Health Grant MH45156, and a Howard Hughes Medical Institute predoctoral fellowship (N.N.U.).

Correspondence should be addressed to German Barrionuevo, 446 Crawford Hall, University of Pittsburgh, Pittsburgh, PA 15260.

Copyright (C) 1996 Society for Neuroscience $0270-6474 / 96 / 164293-07 \$ 05.00 / 0$
}

conditions. Sustained presynaptic activation is sufficient to induce the non-Hebbian form of mossy fiber LTP, whereas brief presynaptic activation coincident with postsynaptic depolarization is required to induce the Hebbian form. We suggest that non-Hebbian forms of plasticity may play an important role in dynamically regulating the thresholds for inducing Hebbian forms of plasticity.

Key words: hippocampus; CA3; LTP; mossy fiber; Hebbian; synaptic plasticity; granule cell; dentate gyrus; NMDA
The reasons for these conflicting results are unclear. Many of these data could be explained by the hypothesis that both Hebbian and non-Hebbian forms of LTP can be induced at the mossy fiber-CA3 synapse. According to this hypothesis, the kind of potentiation observed in a given experiment depends on factors such as the type of LTP-inducing stimulus applied and the recording conditions. Preliminary experiments in our laboratory have suggested that different patterns of high-frequency stimulation (HFS) may induce two types of mossy fiber LTP, each having different time courses and different sensitivities to whole-cell dialysis (Urban et al., 1994; Langdon et al., 1995). Thus, we investigated the requirements for induction of mossy fiber LTP by two different patterns of HFS.

\section{MATERIALS AND METHODS}

Hippocampal slice preparation. Hippocampal slices, 350-500 $\mu \mathrm{m}$ thick, were prepared from 21-35-d-old male Sprague-Dawley rats. Animals were anesthetized deeply and then perfused transcardially with a coldmodified artificial cerebral spinal fluid (M-ACSF) (concentrations in mM: 229.0 sucrose, $1.9 \mathrm{KCl}, 1.2 \mathrm{Na}_{2} \mathrm{PO}_{4} \cdot 7 \mathrm{H}_{2} \mathrm{O}, 25.0 \mathrm{NaHCO}_{3}, 10.0 \mathrm{HEPES}$, 10.0 dextrose, and $10.0 \mathrm{MgCl}_{2}$ ) bubbled with $97.5 \mathrm{O}_{2} / 2.5 \mathrm{CO}_{2}, \mathrm{pH}=7.4$ (Aghajanian and Rasmussen, 1989; Henze et al., 1996). In most experiments, 2-5 $\mu \mathrm{M}$ MK-801 was included in this M-ACSF to improve slice viability. After the perfusion, the animals were decapitated, the brains removed rapidly, and the slices cut on a vibratome. Slices were transferred to an incubation chamber containing normal ACSF (concentrations in mM: $125.0 \mathrm{NaCl}, 2.0 \mathrm{KCl}, 10.0$ dextrose, $26.0 \mathrm{NaHCO}_{3}, 1.0 \mathrm{MgCl}_{2}$, and $2.5 \mathrm{CaCl}_{2}$ ) bubbled with $95 \% \mathrm{O}_{2} / 5 \% \mathrm{CO}_{2}$ at room temperature. Often, MK-801 $(2-10 \mu \mathrm{M})$ was added to the incubation medium to improve slice viability and to ensure complete blockade of NMDA receptors during experiments. After incubation, slices were transferred to the recording chamber and submerged in normal ACSF at $30-34^{\circ} \mathrm{C}$. In experiments in which bicuculline was added to the bathing medium, $\mathrm{CaCl}_{2}$ and $\mathrm{MgCl}_{2}$ concentrations were increased to $3 \mathrm{mM}$ to prevent bursting of CA3 cells.

Stimulation and recording techniques. Extracellular glass electrodes (1-3 $\mathrm{M} \Omega$ ) were filled with $0.5 \mathrm{M} \mathrm{NaCl}$. Intracellular sharp electrodes (50-80 $\mathrm{M} \Omega$ ) were filled with $2 \mathrm{M}$ potassium acetate. Whole-cell patch-clamp electrodes (3-7 M $\Omega$ ) were filled with either a cesium-floride-based pipette solution containing (in mM): $120 \mathrm{CsF}, 20 \mathrm{CsCl}, 1 \mathrm{BAPTA}$, and 10 HEPES or an ATP-containing solution containing (in $\mathrm{mM}$ ): 115 potas- 
sium gluconate, $20 \mathrm{KCl}, 10 \mathrm{HEPES}, 0.6 \mathrm{EGTA}, 10$ sodium phosphocreatine, $4 \mathrm{MgATP}$, and $0.3 \mathrm{GTP}$ plus $50 \mathrm{U} / \mathrm{ml}$ creatine phosphate. Wholecell currents were recorded while holding cells at $-80 \mathrm{mV}$, using techniques described previously (Langdon et al., 1995). Series resistances were $<15 \mathrm{M} \Omega$ and were uncompensated. MK-801 $(10 \mu \mathrm{M})$ was present for the duration of all experiments to block LTP at the CA3 to CA3 collateral synapses. The effectiveness of the blockade of NMDA receptordependent LTP by MK-801 was tested in 10 slices by applying HFS to a stimulating electrode placed in the stratum (s.) radiatum of CA3 while recording nonmossy fiber field EPSPs, also from CA3 s. radiatum. In these cases, LTP was never observed (mean amplitude $=102 \pm 8 \%$ of baseline).

Mossy fiber responses were evoked by using one or two stimulating electrodes placed in the granule cell layer of the dentate gyrus. Test stimuli were administered at $0.1 \mathrm{~Hz}$. In the preliminary experiments shown in Figure 1, $A$ and $B$, HFS consisted of either $6-10$ pulses at 100 $\mathrm{Hz}$ repeated eight times at $5 \mathrm{sec}$ intervals (Jaffe and Johnston, 1990) for brief-HFS (B-HFS) or 100 pulses at $100 \mathrm{~Hz}$ repeated $1-3$ times at an interval of 10-20 sec (Zalutsky and Nicoll, 1990) for long-HFS (L-HFS). In all subsequent experiments, B-HFS consisted of eight pulses at $100 \mathrm{~Hz}$ repeated eight times at $5 \mathrm{sec}$ intervals, and L-HFS consisted of 100 pulses at $100 \mathrm{~Hz}$ repeated three times at a $10 \mathrm{sec}$ interval. The magnitude of LTP was calculated by dividing the average amplitude of 10 responses evoked 15-20 min after the HFS by the average amplitude of responses evoked in the $5 \mathrm{~min}$ before the delivery of the HFS.

Identification of mossy fiber responses. Because of the complicated circuitry of area CA3 (Claiborne et al., 1993), care must be taken to distinguish mossy fiber field EPSPs from population spikes and from nonmossy fiber EPSPs elicited by activation of recurrent collaterals. Following Castillo et al. (1994), a sink (negative) current recorded in the s. lucidum evoked by dentate gyrus stimulation was identified as a mossy fiber field EPSP under the following conditions. (1) The duration of the sink current was $>4 \mathrm{msec}$. Because we have observed antidromically evoked population spikes in area CA 3 to last $2 \mathrm{msec}$ or less, this criterion allows us to distinguish mossy fiber EPSPs from CA3 population spikes. (2) A source rather than sink current was observed when the recording electrode was moved from the s. lucidum into the s. radiatum. This criterion allowed us to distinguish mossy fiber from nonmossy fiber field EPSPs. Further, care was taken to minimize the positivity that sometimes follows the mossy fiber EPSP. This positivity, which may contaminate the mossy fiber field EPSP, may represent activation of collateral synapses. When field EPSPs were measured, the stimulation intensities used elicited a response of $<60 \%$ of a maximal response.

Mossy fiber responses recorded by using intracellular techniques (sharp electrodes or whole-cell recordings) were analyzed if latencies were $<5$ msec and responses had no obvious secondary peaks. Because the interpretation of experiments involving postsynaptic manipulations depends on having an uncontaminated monosynaptic response, in experiments of this sort we selected responses of $<2 \mathrm{mV}$ or $100 \mathrm{pA}$ average peak amplitude and did not include GABA antagonists in the bathing solution. Under these conditions, we have observed very little polysynaptic contamination of mossy fiber responses (Langdon et al., 1993). Because complicated waveforms may be typical of mossy fiber synaptic responses elicited by bulk stimulation (Langdon et al., 1993), we did not use criteria based on waveform kinetics (Claiborne et al., 1993).

Measurements of mossy fiber field responses. Field EPSP amplitudes were calculated after subtracting the mossy fiber volley from the evoked response. The mossy fiber volley was recorded at the end of the experiment after blocking synaptic transmission with $0 \mathrm{mM} \mathrm{Ca}^{2+}$ and $6 \mathrm{mM}$ $\mathrm{Mg}^{2+}$ or $10 \mu \mathrm{M}$ CNQX. All results are given as mean \pm SEM. Waveforms shown are averages of 3-6 consecutive responses. All drugs were purchased from Sigma (St. Louis, MO), with the exception of APV and MK-801 (Research Biochemicals, Natick, MA).

\section{RESULTS}

\section{Preliminary studies}

To ensure that the extracellular field potential responses measured by using the criteria described above reflected the strength of mossy fiber synaptic transmission, we tested the effect of manipulations of synaptic and nonsynaptic currents on the amplitude of EPSPs. While recording a presumed mossy fiber field EPSP, we increased the extracellular calcium from 2.5 to $4.0 \mathrm{~mm}$. In all cases ( $3 / 3$ slices), this manipulation increased the amplitude
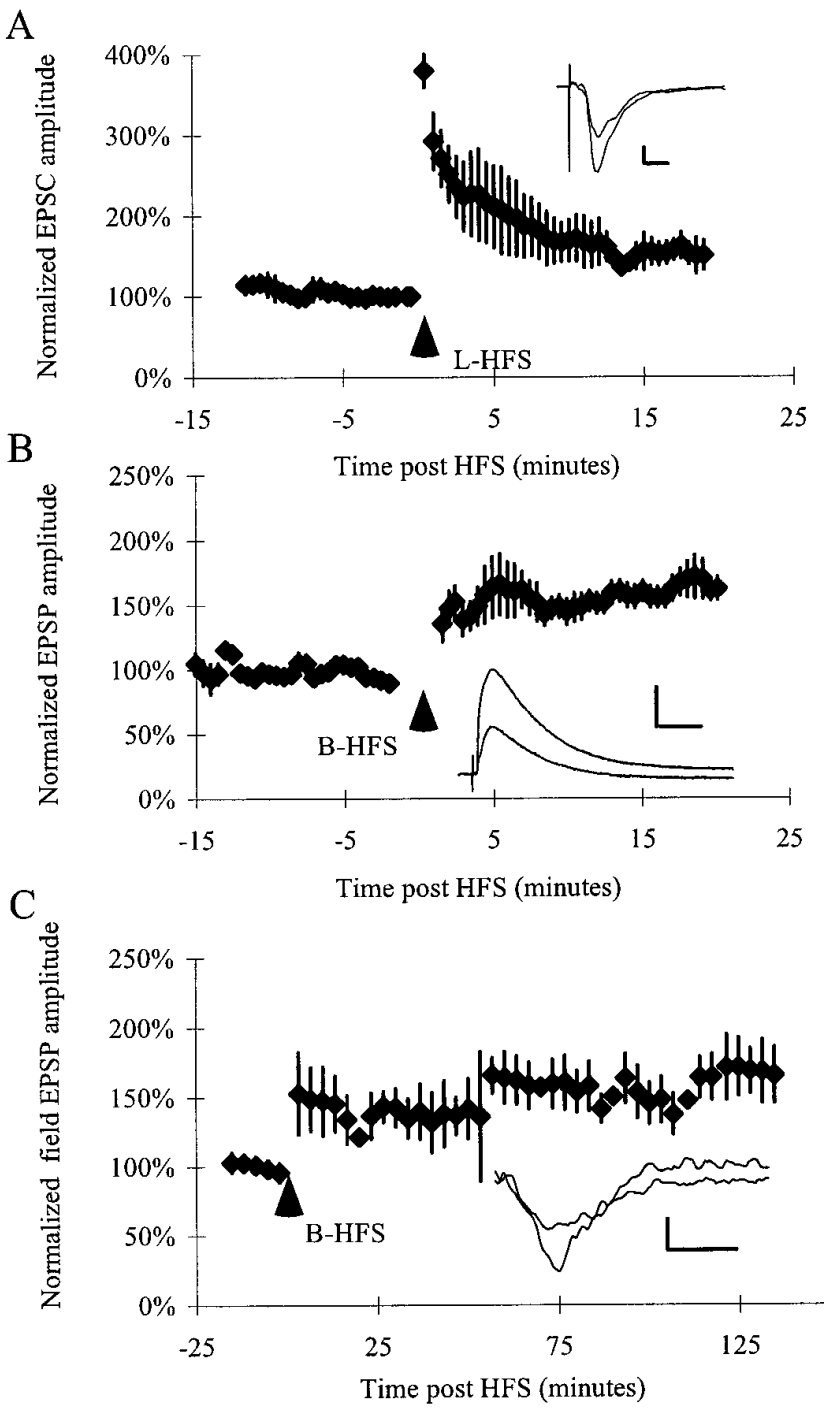

Figure 1. L-HFS and B-HFS result in different time courses of potentiation. Time of HFS is indicated by the triangle. Each point represents the peak amplitude at the given time point from seven experiments, normalized and averaged. Error bars give the SE. Sample waveforms from before and $15 \mathrm{~min}$ after HFS are shown in the inset. $A$, In seven whole-cell recordings, $L-H F S$ to the mossy fibers resulted in very large PTP lasting for several min after HFS, followed by stable LTP $(151 \pm 18 \%$ of control; $n=7$ ). Scale bar: $100 \mathrm{pA}, 10 \mathrm{msec}$. $B$, In seven experiments, EPSPs were recorded with intracellular sharp electrodes. $B$-HFS to the mossy fibers resulted in LTP ( $169 \pm 11 \%$ of control; $n=7)$ without PTP. Scale bar: 10 $\mathrm{mV}, 20 \mathrm{msec}$. $C$, In three experiments, potentiation of the extracellularly recorded field EPSP lasted $>2.5 \mathrm{hr}$ after B-HFS. The potentiation at 150 min was $165 \pm 20 \%$ of control. Scale bar: $0.1 \mathrm{mV}, 5 \mathrm{msec}$.

of the field EPSP. In contrast, addition of up to $5 \mu \mathrm{M}$ bicuculline increased the positivity after the EPSP but had no effect on the measured peak EPSP amplitude, indicating that our measurement of field EPSP amplitude was insensitive to moderate changes in inhibition.

\section{Different patterns of HFS result in different time courses of mossy fiber LTP}

Mossy fiber LTP was induced by using two different HFS protocols. Bicuculline $(10 \mu \mathrm{M})$ was present in the bathing medium to eliminate GABA $_{\mathrm{A}}$ IPSPs/IPSCs and to ensure that any potentia- 
tion observed did not result from a decrease in inhibition. To ensure that postsynaptic depolarization would be sufficient to induce a Hebbian form of LTP (Jaffe and Johnston, 1990), we adjusted the stimulation intensity to give EPSPs of $>5 \mathrm{mV}$ in amplitude (measured in current clamp). All cells fired action potentials in response to HFS. L-HFS induced LTP $(151 \pm 18 \%$; $n=7$; Fig. $1 A$ ) of mossy fiber EPSCs recorded with whole-cell electrodes. B-HFS induced a similar amount of potentiation (170 $\pm 11 \% ; n=7$; Fig. $1 B$ ) when mossy fiber EPSPs were recorded with intracellular sharp electrodes.

Although B-HFS and L-HFS both induced stable LTP of similar magnitude, the time course of the potentiation induced by these two patterns of HFS differed greatly. As has been observed previously (Langdon et al., 1995), L-HFS induced an extremely large, long-lasting post-tetanic potentiation (PTP) that decayed to stable LTP. In contrast, B-HFS induced almost no PTP, and the potentiation that followed increased for $>20 \mathrm{~min}$. Also, a similar time course was observed in experiments in which LTP of the field EPSP was followed for $>130 \mathrm{~min}$ after B-HFS (Fig. 1C).

Because these two patterns of HFS induced LTP of such markedly different time courses, we next tested the requirements for induction of LTP by these two patterns of HFS.

\section{Induction of mossy fiber LTP by B-HFS depends on stimulation intensity}

Increasing stimulation intensity increases the number of activated axons, thus resulting in greater postsynaptic depolarization. By examining the effect of increasing stimulation strength on the magnitude of LTP induced, we tested whether induction of mossy fiber LTP by B-HFS depends on postsynaptic depolarization. Previously, induction of mossy fiber LTP by L-HFS has been shown to be independent of postsynaptic depolarization (Ito and Sugiyama, 1991; Castillo et al., 1994; Langdon et al., 1995).

Mossy fiber field EPSPs were evoked by using a stimulation intensity that resulted in a response of $<30 \%$ of maximal. Based on preliminary experiments and on results in vivo (Derrick and Martinez, 1994a), we expected that such low intensity stimulation would be insufficient to induce Hebbian mossy fiber LTP. After recording $15 \mathrm{~min}$ of baseline, we administered B-HFS at this stimulation intensity. This HFS resulted in no significant LTP (amplitude $=98 \pm 6 \% ; n=6$; Fig. $2 B$ ). Then we increased the stimulation intensity by an amount that increased response amplitude to $>50 \%$ of maximal. After recording an additional baseline period, we again applied B-HFS. In each case, this second application of B-HFS resulted in significantly more LTP than the first $(137 \pm 9 \% ; n=6 ; p<0.02$, paired $t$ test; Fig. $2 B)$, suggesting that the induction of LTP by B-HFS depends on the magnitude of postsynaptic depolarization caused by the inducing stimulus.

\section{Induction of mossy fiber LTP by B-HFS is blocked by kynurenic acid}

A more direct test of the hypothesis that postsynaptic depolarization is required for the induction of LTP involves blocking postsynaptic responses without changing the presynaptic activation. If induction of LTP by B-HFS depends on the postsynaptic depolarization mediated by AMPA receptor activation, then blockade of NMDA and AMPA receptors by $10 \mathrm{~mm}$ kynurenic acid (KYN) during the tetanus should prevent LTP induction.

After recording stable baseline responses to dentate gyrus stimulation, KYN was applied until the field EPSP was eliminated. Then we administered B-HFS, L-HFS, or no HFS (control) to the pathway, followed by washout of the KYN. In control experiments

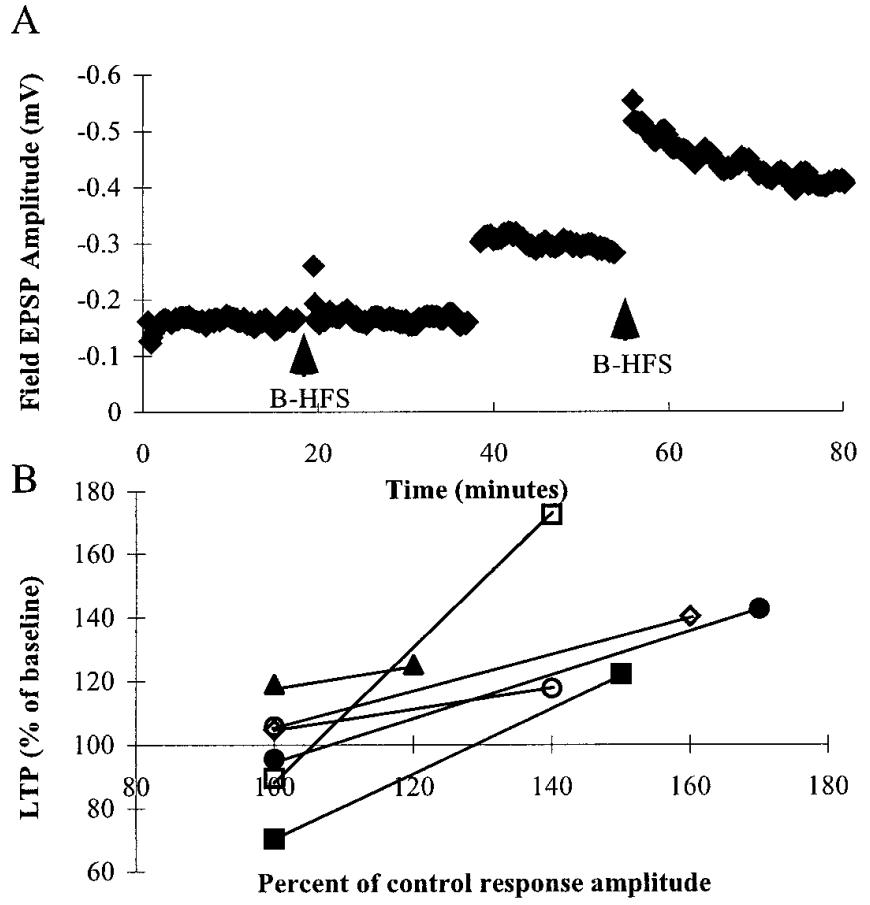

Figure 2. Induction of mossy fiber LTP by B-HFS depends on stimulation intensity. $A$, Mossy fiber field EPSPs were recorded in response to stimulation of the granule cells of the dentate gyrus. $B$-HFS was applied at the indicated times. Initial responses were recorded by using a stimulation intensity that elicited a response $<30 \%$ of maximal amplitude. Application of $B-H F S$ at this stimulation intensity did not induce LTP. A second application of $B$-HFS at a higher intensity resulted in LTP. $B$, Data from six experiments showing results similar to the experiment in $A$. Each pair of symbols with a connecting line represents data from one slice. When applied at a stimulation intensity $<30 \%$ of maximal, B-HFS failed to induce potentiation (post-B-HFS $=98 \pm 7 \%$ of control; $n=6$ ). Symbols clustered at the left end of each line indicate the amount of LTP observed at $20 \mathrm{~min}$ after this first B-HFS. When the stimulation intensity was increased by the factor indicated on the $x$-axis, the same B-HFS induced significant LTP (post-B-HFS $=137 \pm 9 \%$ of control; $p<0.02$ ).

(no HFS), KYN washed out in $\sim 35 \mathrm{~min}$. Therefore, induction of LTP was evaluated by comparing the amplitude of evoked EPSPs before and 35 min after application of the KYN.

When L-HFS was applied in the presence of KYN, we observed large potentiation after washout of the KYN (L-HFS $=194 \pm$ $29 \%$ of control; Fig. $3 A$ ), confirming previous results (Ito and Sugiyama, 1991; Castillo et al., 1994). In contrast, applying B-HFS under the same conditions resulted in no potentiation at $35 \mathrm{~min}$ after washout of the antagonist (B-HFS $=92 \pm 7 \%$ of baseline; $n=15$; Fig. $3 B$ ). In 10 of these 15 slices, we stimulated a second, independent mossy fiber pathway that served as a control and did not receive HFS. In these experiments, at $35 \mathrm{~min}$ after washout of the KYN, the EPSP amplitude in both pathways had recovered equally (B-HFS $=90 \pm 8 \%$; no HFS $=87 \pm 6 \%$ baseline; $n=10$; $p>0.2$, paired $t$ test; Fig. $3 B$ ).

In 12 of the 15 experiments in which B-HFS failed to induce LTP in the presence of KYN, a second B-HFS was applied after washout of KYN and did induce LTP $(161 \pm 25 \%$ of baseline; $n=$ 12; Fig. $3 B$ ). In the remaining three experiments, L-HFS was applied after washout of KYN and also induced LTP (193 $\pm 54 \%$ of control; $n=3$; Fig. $3 B$ ). 
A
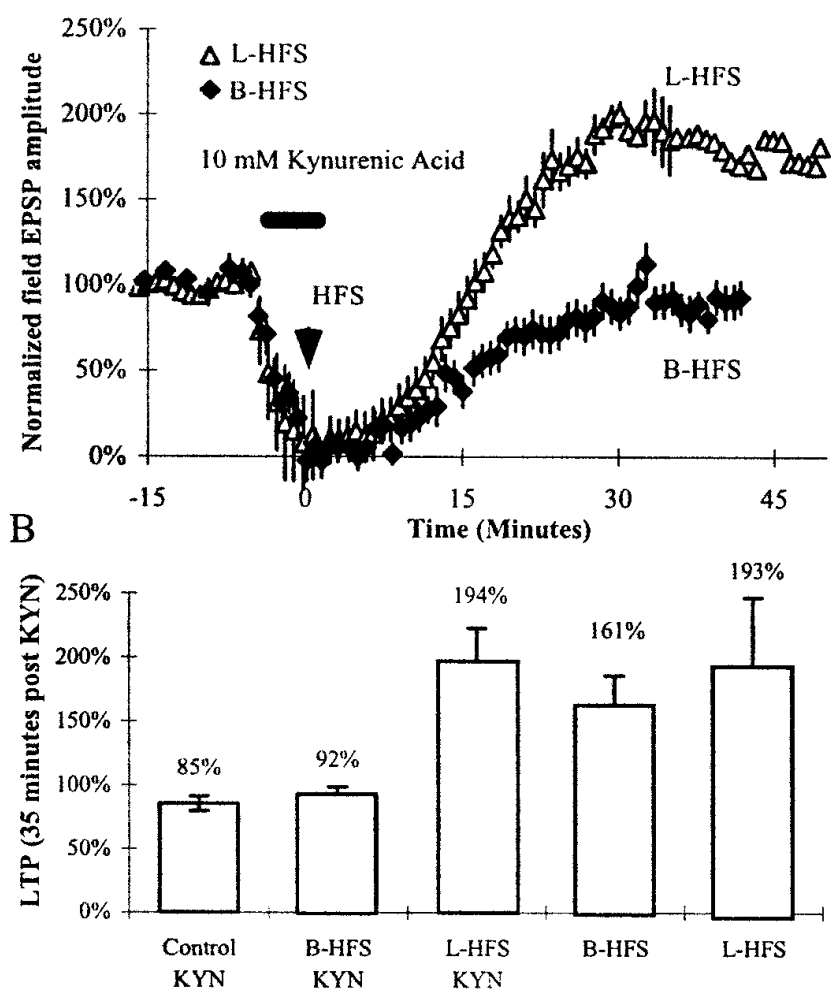

Figure 3. Induction of mossy fiber LTP by B-HFS requires glutamatergic ionotropic synaptic transmission. $A$, After recording baseline field EPSPs, 10 mM kynurenic acid (KYN) was applied to block AMPA and NMDA receptors. When evoked EPSPs were eliminated, B-HFS (black diamonds) or L-HFS (open triangles) was applied, and the KYN was washed out. After L-HFS, the response recovered to a potentiated level (response at 35 min post-HFS $=194 \pm 29 \%$ of baseline; $n=3$ ), whereas after B-HFS, the response was not potentiated (response at 35 min post-HFS $=92 \pm$ $7 \%$ of baseline; $n=15$ ). $B$, Group data showing recovery from KYN after no HFS (control), B-HFS, and $L-H F S$ were applied during the KYN. Also shown, data from experiments in which LTP was induced after washout of KYN by either $B$-HFS or $L-H F S$ in the 15 slices in which B-HFS first was applied in the presence of KYN.

\section{Induction of LTP by B-HFS is blocked by whole-cell dialysis}

Next we performed experiments to test the role of postsynaptic activation in the induction of mossy fiber LTP by B-HFS and L-HFS at the single-cell level. Whole-cell (voltage-clamp) recordings were made using electrodes containing BAPTA and cesium fluoride to buffer postsynaptic calcium (see Materials and Methods). During the HFS, the cell was held at $-60 \mathrm{mV}$ in current clamp, and in all cases the cells fired action potentials in response to the HFS. To favor postsynaptic depolarization and induction of Hebbian LTP, we performed initial experiments in the presence of $10 \mu \mathrm{M}$ bicuculline and used stimulation intensities that gave EPSP amplitudes of $>5 \mathrm{mV}$ when measured in current clamp. Under these conditions, B-HFS failed to induce mossy fiber LTP, whereas in the eight cells tested, subsequent L-HFS induced significantly more potentiation (B-HFS $=102 \pm 20 \%$ of baseline, $n=9$, vs L-HFS $=154 \pm 17 \%$ of baseline, $n=8 ; p<0.05$ ). In contrast, when cells were recorded with sharp electrodes under these same conditions (in current clamp), B-HFS reliably induced LTP $(157 \pm 21 \%$ of baseline, $n=11)$. We take these data to indicate that postsynaptic dialysis and calcium buffering via the whole-cell recording electrode blocked induction of LTP by B-HFS but not by L-HFS.

In three additional experiments, EPSCs were recorded with whole-cell electrodes filled with a solution containing an ATPregenerating system (see Materials and Methods). This solution supports NMDA receptor-dependent LTP in CA3 pyramidal cells for up to 30 min after break-in (N. N. Urban and G. Barrionuevo, unpublished observations). B-HFS was applied $\sim 10 \mathrm{~min}$ after whole-cell break-in while depolarizing the cells to $-30 \mathrm{mV}$ in current clamp. In none of these three cases was LTP induced (amplitude at 20 min post-HFS $=97 \pm 8 \%$ of baseline), indicating that whole-cell dialysis with minimal calcium buffering is sufficient to block rapidly the induction of mossy fiber LTP by B-HFS.

Although suggestive, these results were unsatisfying because of two problems. First, in these experiments, we did not know whether B-HFS induced mossy fiber LTP in these slices at CA3 cells that were not subject to postsynaptic manipulations. Second, both the presence of bicuculline and the use of high stimulation intensities favor contamination of responses by polysynaptic, nonmossy fiber EPSCs. Thus, any potentiation that we observed may have resulted from the recruitment of additional CA3 pyramidal cells synapsing onto the subject cell. Such potentiation of a polysynaptic input would be unaffected by the postsynaptic manipulations described above (Johnston et al., 1992).

In subsequent experiments, we sought to address these problems. By simultaneously recording mossy fiber EPSCs and field EPSPs, we ensured that LTP was occurring at synapses elsewhere in the slice. By omitting bicuculline and adjusting stimulation intensity to evoke responses of $<100 \mathrm{pA}$ or $1 \mathrm{mV}$ in the cell being recorded, we reduced the likelihood of polysynaptic contamination. In these experiments, the recording medium contained 25 $\mu \mathrm{M}$ D-APV in addition to $10 \mu \mathrm{M}$ MK-801. Under these conditions, B-HFS induced significant LTP of the field EPSP but never induced LTP of the whole-cell recorded EPSC (averages at $20 \mathrm{~min}$ after the HFS: EPSP $=162 \pm 9 \%$ of baseline; EPSC $=94 \pm 4 \%$ of baseline; $n=5 ; p<0.02$, paired $t$ test; Fig. $4 A$ ). In the four of these five cases in which the recording lasted long enough, subsequent L-HFS resulted in induction of mossy fiber LTP of both the whole-cell EPSC and the field EPSP.

\section{Induction of mossy fiber LTP by B-HFS depends on postsynaptic depolarization}

In a final set of experiments, we tested directly whether induction of mossy fiber LTP by B-HFS requires coincident postsynaptic depolarization and presynaptic activation. In these experiments, intracellular recordings from $\mathrm{CA} 3$ pyramidal cells were obtained with sharp electrodes. Because we were testing the sensitivity of LTP induction to a postsynaptic manipulation (see Materials and Methods), stimulation intensities resulting in small amplitude $(<2$ $\mathrm{mV}$ ) EPSPs were used, and inhibition was not blocked. After recording a stable baseline, we applied B-HFS simultaneously with a $2.0-2.5 \mathrm{nA}$ hyperpolarizing current of $100 \mathrm{msec}$ duration. During this manipulation, the postsynaptic cell never fired action potentials. At $15 \mathrm{~min}$ after this manipulation, the amplitude of evoked EPSPs did not differ from that of control $(91 \pm 11 \% ; n=$ 6; Fig. 5A). Then we injected a $2.0-2.5 \mathrm{nA}$ depolarizing current pulse, also of $100 \mathrm{msec}$ duration, without presynaptic stimulation. This manipulation also failed to induce LTP $(81 \pm 14 \% ; n=6$; Fig. $5 B$ ). However, similar intracellular depolarization paired with B-HFS induced significant LTP in these same cells $(197 \pm 32 \%$; $n=6$; Fig. $5 C$ ). 


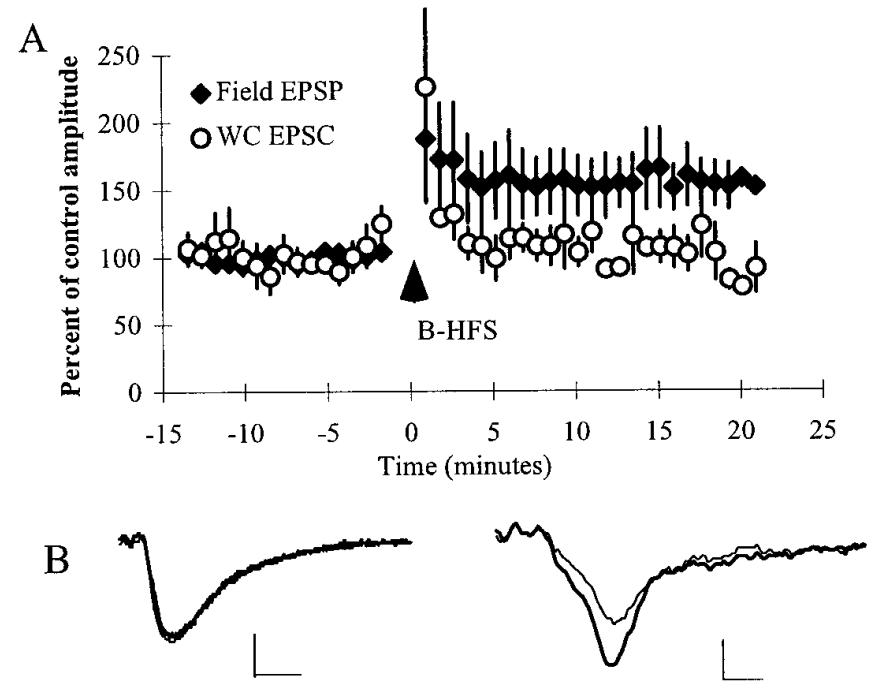

Figure 4. B-HFS does not induce mossy fiber LTP after whole-cell dialysis. $A$, Averaged data from five experiments in which whole-cell EPSCs (WC EPSC) and field EPSPs (Field EPSP) were recorded simultaneously. At 20 min post-B-HFS, the field EPSP, but not the whole-cell EPSC, was potentiated (EPSP $=162 \pm 9 \%$; EPSC $=94 \pm 20 \% ; n=5$, $p<0.02)$. B, EPSC and field EPSP waveforms from one example experiment. Scale bars: $5 \mathrm{msec}, 25 \mathrm{pA}(E P S C) ; 2 \mathrm{msec}, 0.1 \mathrm{mV}(E P S P)$.

\section{DISCUSSION}

\section{Summary}

By comparing the requirements for LTP induction by two different patterns of HFS, we have tested the hypothesis that both Hebbian and non-Hebbian forms of LTP can be induced at the mossy fiber CA3 synapse.
Data from a number of experiments (Zalutsky and Nicoll, 1990; Ito and Sugiyama, 1991; Katsuki et al., 1991; Castillo et al., 1994; Langdon et al., 1995), including some described above, demonstrate that mossy fiber LTP may be induced using L-HFS under conditions that prevent depolarization of and calcium elevation in the postsynaptic cell. Thus, L-HFS can induce a non-Hebbian form of mossy fiber LTP. In the present study, we show that induction of mossy fiber LTP by B-HFS requires postsynaptic depolarization to be paired with presynaptic stimulation (Jaffe and Johnston, 1990). Also, we have demonstrated that postsynaptic manipulations block induction of mossy fiber LTP by B-HFS in a single cell, even when potentiation is observed at other CA3 cells. Together these data indicate that both non-Hebbian and Hebbian LTP can be induced at the same synapse.

\section{Differences between B-HFS and L-HFS}

The conclusion that these two patterns of HFS induce different forms of LTP leads to the question of how these two stimulation protocols differently affect pre- and postsynaptic activity. In current-clamp experiments, both of these patterns of HFS normally result in repetitive spiking of the postsynaptic cell, but only L-HFS induces mossy fiber LTP when this spiking is blocked. Because L-HFS can cause high levels of postsynaptic depolarization and action potential firing, we would predict that if the non-Hebbian form of mossy fiber LTP could be blocked selectively, L-HFS still would induce the Hebbian form of mossy fiber LTP. In contrast, we have shown that B-HFS fails to induce the non-Hebbian form of mossy fiber LTP when induction of the Hebbian form is prevented. We hypothesize that the ineffectiveness of B-HFS at inducing the non-Hebbian form of mossy fiber LTP likely is attributable to insufficient elevation of presynaptic calcium. Calcium-imaging data from mossy fiber presynaptic terminals (Regehr et al., 1994) indicate
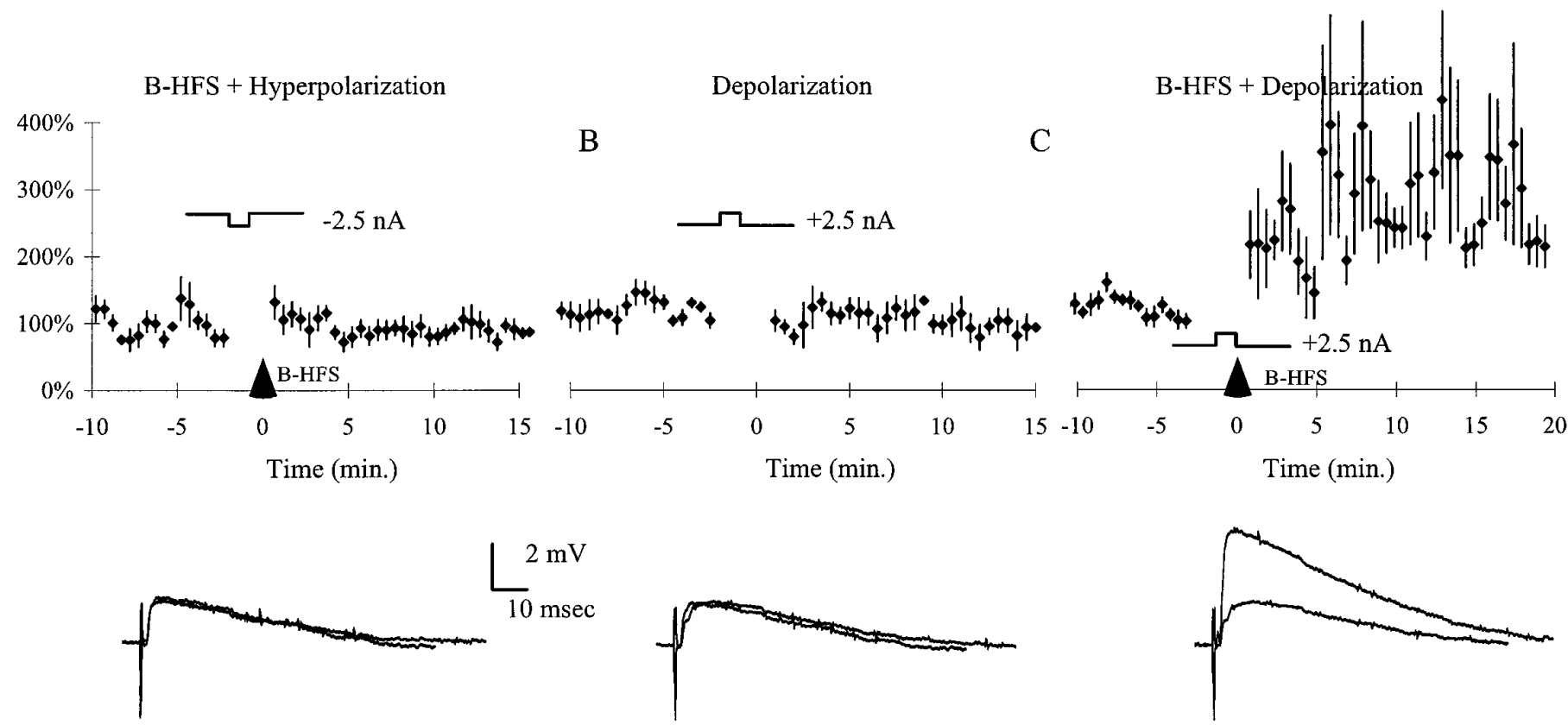

Figure 5. Induction of mossy fiber LTP by B-HFS requires simultaneous afferent stimulation and postsynaptic depolarization. In six cells, three separate manipulations were tested for their effect on mossy fiber EPSP amplitude. $A$, B-HFS paired with hyperpolarization $(-2.5 \mathrm{nA}$, $100 \mathrm{msec}$ square pulses passed simultaneously with the application of the B-HFS) failed to induce long-lasting changes in mossy fiber EPSP amplitude (amplitude at 15 min $=$ $91 \pm 11 \%$ of baseline). $B$, Depolarization alone $(+2.5 \mathrm{nA}, 100$ msec square pulse repeated 10 times at 5 sec intervals) resulted in a slight decrease in mossy fiber EPSP amplitude (amplitude at $15 \mathrm{~min}=81 \pm 14 \%$ of baseline). $C$, Pairing of depolarization ( $+2.5 \mathrm{nA}, 100 \mathrm{msec}$ square pulse) with the B-HFS resulted in LTP (amplitude at $15 \mathrm{~min}=197 \pm 32 \%$ of control). 
that L-HFS results in a several-fold greater elevation of presynaptic calcium than B-HFS. This high level of presynaptic calcium may be required to activate the second-messenger systems (Huang et al., 1994; Weisskopf et al., 1994) that are responsible for the changes observed in the non-Hebbian form of mossy fiber LTP.

\section{Functional implications}

Using computational models, the properties of neural networks in which synapses are modified according to Hebbian rules have been well studied (Kohonen, 1984). However, the functional significance of the various sorts of long- and short-term nonHebbian forms of plasticity (Alonso et al., 1990; Bradler and Barrionuevo, 1990; Kullman et al., 1992; Wyllie and Nicoll, 1994) are not well understood. Moreover, the nonassociative induction requirements of non-Hebbian plasticity raise questions about the significance of non-Hebbian synaptic modification in the specific storage of information by the brain. Here we propose that one important function of non-Hebbian plasticity may be to regulate the induction of Hebbian forms of plasticity by dynamically adjusting the threshold for LTP induction.

Hebbian LTP is induced when pre- and postsynaptic activity simultaneously exceed certain thresholds. The non-Hebbian potentiation of a synapse between two cells will increase the amount of postsynaptic depolarization resulting from each presynaptic action potential. Thus, a non-Hebbian potentiation at a given synapse will increase the probability that, for subsequent stimuli, the pre- and postsynaptic thresholds for Hebbian potentiation of this synapse will be met simultaneously. Such a process could be thought of as a way of increasing the time window in which stimuli must occur to result in the induction of Hebbian LTP.

The very large initial PTP that is associated with non-Hebbian mossy fiber LTP is well suited for such a mechanism of threshold modification. For several minutes after L-HFS, mossy fiber synaptic responses are potentiated several-fold (see Fig. 1A) (Langdon et al., 1995). This large potentiation would make these synapses extremely effective at depolarizing the postsynaptic CA3 cell. Thus, these synapses would be temporarily "sensitized" to associative Hebbian modification. Such a mechanism may be especially important when CA3 cells are subject to high levels of inhibition, for example when an animal is exploring a familiar environment (Wilson and McNaughton, 1993). During these periods, CA3 cell activity is low, and thus the probability of Hebbian potentiation is likely to be reduced.

Network models have shown that systems with both transient and persistent forms of synaptic modification potentiation have greater storage capacities and are better able to adapt to different short-term contexts while avoiding interference with long-term storage (Gardner-Medwin, 1989). In other models, transient forms of potentiation have proven useful for the encoding of sequences of stimuli (Granger et al., 1994; Buonomano and Merzenich, 1995). Similar "sequence-encoding" functions for CA3 have been suggested, based on similarities between the CA3 anatomy and the architecture of neural network models with recurrent connections (Churchland and Sejnowski, 1992). These observations lead us to hypothesize that both the plasticity in and anatomy of the CA3 network may allow this region of the hippocampus to process and store sequences of inputs efficiently.

\section{REFERENCES}

Aghajanian GK, Rasmussen K (1989) Intracellular studies in the facial nucleus illustrating a simple new method for obtaining viable motoneurons in adult rat brain slices. Synapse 3:331-338.

Alonso A, De Curtis M, Llinás R (1990) Postsynaptic Hebbian and non-Hebbian long-term potentiation of synaptic efficacy in the entorhinal cortex in slices and in the isolated adult guinea pig brain. Proc Natl Acad Sci USA 87:9280-9284.

Bliss TVP, Collingridge GL (1993) A synaptic model of memory: longterm potentiation in the hippocampus. Nature 361:31-39.

Bradler JE, Barrionuevo G (1990) Heterosynaptic correlates of longterm potentiation induction in hippocampal CA3 neurons. Neuroscience 35:265-271.

Bramham CR (1992) Opoid receptor-dependent long-term potentiation: peptidergic regulation of synaptic plasticity in the hippocampus. Neurochem Int 20:441-455.

Buonomano DV, Merzenich MM (1995) Temporal information transformed into a spatial code by a neural network with realistic properties. Science 267:1028-1030.

Castillo PE, Weisskopf MG, Nicoll RA (1994) The role of calcium channels in hippocampal mossy fiber synaptic transmission and long-term potentiation. Neuron 12:261-269.

Churchland PA, Sejnowski TJ (1992) The computational brain, pp 115125. Cambridge, MA: MIT.

Claiborne BJ, Xiang Z, Brown TH (1993) Hippocampal circuitry complicates analysis of long-term potentiation in mossy fiber synapses. Hippocampus 3:115-122.

Derrick BE, Martinez Jr JL (1994a) Frequency-dependent associative long-term potentiation at the hippocampal mossy fiber-CA3 synapse. Proc Natl Acad Sci USA 91:10290-10294.

Derrick BE, Martinez Jr JL (1994b) Opoid receptor activation is one factor underlying the frequency dependence of mossy fiber LTP induction. J Neurosci 14:4359-4367.

Gardner-Medwin AR (1989) Doubly modifiable synapses: a model of short and long term auto-associative memory. Proc R Soc Lond [Biol] 238:137-154.

Granger R, Whitson J, Larson J, Lynch G (1994) Non-Hebbian properties of long-term potentiation enable high-capacity encoding of temporal sequences. Proc Natl Acad Sci USA 91:10104-10108.

Grover LM, Teyler TJ (1992) $N$-methyl-D-aspartate receptor-independent long-term potentiation in area CA1 of rat hippocampus: input specific induction and preclusion in a non-tetanized pathway. Neuroscience 49:7-11.

Harris EW, Cotman CW (1986) Long-term potentiation of guinea pig mossy fiber responses is not blocked by $N$-methyl-D-aspartate antagonists. Neurosci Lett 70:132-137.

Hebb DO (1949) The organization of behavior, p 62. New York: Wiley.

Henze DA, Cameron WE, Barrionuevo G (1996) Dendritic morphology and its effects on the amplitude and rise time of synaptic signals in hippocampal CA3 pyramidal cells. J Comp Neurol 369:331-344.

Huang Y-Y, Li X-C, Kandel ER (1994) cAMP contributes to mossy fiber LTP by initiating both a covalently mediated early phase and a macromolecular synthesis-dependent late phase. Cell 79:69-79.

Ito I, Sugiyama H (1991) Roles of glutamate receptors in long-term potentiation at hippocampal mossy fiber synapses. NeuroReport 2:333-336.

Jaffe D, Johnston D (1990) Induction of long-term potentiation at hippocampal mossy fiber synapses follows a Hebbian rule. J Neurophysiol 64:948-960.

Johnston D, Williams S, Jaffe D, Gray R (1992) NMDA-receptorindependent long-term potentiation. Annu Rev Physiol 54:489-505.

Katsuki H, Kaneko S, Tajima A, Satoh M (1991) Separate mechanisms of long-term potentiation in two input systems to CA3 pyramidal neurons of rat hippocampal slices as revealed by the whole-cell patchclamp technique. Neurosci Res 12:393-402.

Kohonen T (1984) Self-organization and adaptive memory. Berlin: Springer.

Kullman DM, Perkel DJ, Manabe T, Nicoll RA (1992) $\mathrm{Ca}^{2+}$ entry into postsynaptic voltage-sensitive $\mathrm{Ca}^{2+}$ channels can transiently potentiate excitatory synaptic transmission in the hippocampus. Neuron 9:1175-1183.

Langdon RB, Johnson JW, Barrionuevo G (1993) Asynchrony of mossy fiber inputs and excitatory postsynaptic currents in rat hippocampus. J Physiol (Lond) 472:157-176. 
Langdon RB, Johnson JW, Barrionuevo G (1995) Posttetanic potentiation and presynaptically induced long-term potentiation at the mossy fiber synapse in the rat hippocampus. J Neurobiol 26:370-385.

McNaughton BL, Barnes CA (1990) From cooperative synaptic enhancement to associative memory: bridging the abyss. Semin Neurosci 2:403-416.

Nicoll RA, Malenka RC (1995) Contrasting properties of two forms of long-term potentiation in the hippocampus. Nature 377:115-118.

Regehr WG, Delaney KR, Tank DW (1994) The role of presynaptic calcium in short-term enhancement at the hippocampal mossy fiber synapse. J Neurosci 14:523-537.

Weisskopf MG, Castillo PE, Zalutsky RA, Nicoll RA (1994) Mediation of hippocampal mossy fiber long-term potentiation by cyclic AMP. Science 265:1878-1882.

Wilson MA, McNaughton BL (1993) Dynamics of the hippocampal ensemble code for space. Science 261:1055-1058.

Wyllie DJA, Nicoll RA (1994) A role for protein kinases and phosphatases in the $\mathrm{Ca}^{2+}$-induced enhancement of hippocampal AMPA receptormediated synaptic responses. Neuron 13:635-643.

Urban NN, Langdon RB, Barrionuevo G (1994) The duration and pattern of high frequency stimulation influence the time course of potentiation and the mossy fiber-CA3 synapse. Soc Neurosci Abstr 20:715.

Zalutsky RA, Nicoll RA (1990) Comparison of two forms of long-term potentiation in single hippocampal neurons. Science 248:1619-1624. 